\title{
Urine collection practices in a small rural hospital: Evaluation of alignment with antimicrobial stewardship guidelines
}

\section{Corresponding author:} \\ Jennifer Irwin, RN, Infection Control Practitioner \\ Vancouver Coastal Health Authority \\ Powell River General Hospital \\ 5000 Joyce Ave. \\ Powell River, BC V8A 5R3 Canada \\ Tel.: 604-485-3214 \\ Fax: 604-485-3245 \\ jennifer.irwin@vch.ca
}

Jennifer Irwin, RN; ${ }^{1}$ Leslie Forrester, BA (Hons), MA, MSc ${ }^{2}$

${ }^{1}$ Infection Control Practitioner, Vancouver Coastal Health Authority, Powell River General Hospital, Powell River, BC, Canada

${ }^{2}$ Regional Director Data Analytics, Vancouver Coastal Health Authority, Powell River General Hospital, BC, Canada

\begin{abstract}
Background: The diagnosis of a symptomatic urinary tract infection (UTI) can be challenging among elderly patients, resulting in an increased risk for specimen collection and treatment of asymptomatic patients. The Vancouver Coastal Health Authority's Antimicrobial Stewardship Guidelines provide a systematic approach to support clinicians in the assessment and treatment of UTIs. The purpose of this quality review was to evaluate if urine collection practices were in alignment with the guidelines.

Methods: The review involved a retrospective examination of all urine specimens collected from medical and surgical patients of a small rural hospital from September 1, 2015 to August 31, 2016. A survey of staff was also conducted to evaluate the interdisciplinary team's knowledge of urine collection and interpretation and to assess concordance with the guidelines.

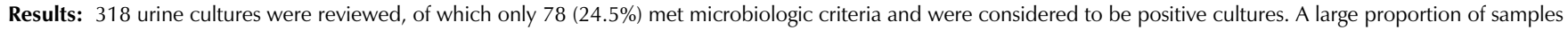
resulted in mixed organisms. There was a highly statistically significant relationship between urinalysis and a positive culture (Fisher's exact test $p<0.0001)$. A positive urine culture (> 100 million CFU/L) was statistically significantly more likely to be observed when there was a positive urinalysis.

Conclusions: Older adults represent a large and growing population of hospitalized patients. Diagnosis of a symptomatic UTI in the elderly can pose challenges. Urine cultures are frequently obtained and a high proportion sent for culturing with missing or negative urinalysis. Urinalysis results are pertinent in the diagnosis of a UTI as there is correlation between a positive urinalysis and a positive urine culture. Adoption of a systematic approach in the management of UTIs would result in consistent and appropriate assessment and treatment of UTIs for elderly patients.
\end{abstract}

\section{KEYWORDS}

Elderly; urinary tract infections; urinalysis; antimicrobial stewardship; systematic approach

\section{INTRODUCTION}

Urinary tract infections (UTIs) have been identified as the single most common healthcare-acquired infection, accounting for $40 \%$ of all hospital-acquired infections and resulting in a substantial burden for patients and the healthcare system [1-3]. UTIs impact patient morbidity and mortality, which can lead to delirium, falls, and an increased length of stay in hospital $[4,5]$.

Older adults are at a greater risk for UTIs due to increased post-void residual volume, prostatic hypertrophy, age-associated changes in immune function, and a growing number of comorbidities [4, 6-8]. Hospitalized older adults generally have more functional impairments, higher cognitive deficits, and a greater number of medical comorbidities. Medical comorbidities, such a stroke and dementia, may predispose individuals to bowel and bladder incontinence, which has been associated with symptomatic UTIs and persistent asymptomatic bacteriuria [6]. However, the most significant risk factor associated with UTIs in hospitalized patients is the presence of a urinary catheter. Approximately $80 \%$ of healthcare-acquired UTIs are catheter-associated [1, 4, 8].

The diagnosis of a symptomatic UTI requires both the presence of genitourinary symptoms in addition to a positive urine culture $[2,6]$. Elderly patients who suffer from significant cognitive deficits that impair their ability to communicate and chronic genitourinary symptoms (e.g., incontinence, urgency, and frequency) make the diagnosis of symptomatic UTIs 


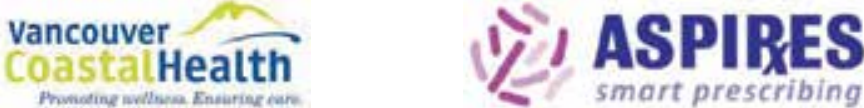

Antimicrobial Stewardship Programme: Innovation, Research, Education, and Safety Quality and Patient Safety, Vancouver Coastal Health Vancouver General Hospital

855 West $12^{\text {th }}$ Avenue, Vancouver, BC, V5Z1M9, Canada

\section{VCH Management of Urinary Tract Infections (UTI) in Non-pregnant Adults}

KEY POINTS:

1. Malodorous/cloudy urine alone is NOT a sign/symptom of UTI and is NOT an indication to obtain urine cultures ${ }^{(1)}$

2. Changes in cognitive function and activities of daily living REQUIRE clinical assessment; never assume these are due to UTI

3. Urine should ALWAYS be collected midstream, by in/out catheterization, or through a new catheter (unless contraindicated) (2)

4. Positive urine cultures in asymptomatic patients should NOT be treated except in pregnancy or prior to urologic/gynecologic surgery

Signs and Symptoms of Suspected UTI (3)

-One of the following in febrile patients (oral temperature $>37.8^{\circ} \mathrm{C}$ [or $1^{\circ} \mathrm{C}$ above baseline in Spinal Cord Injury]) or two of the following in afebrile patients:
i. Acute dysuria
vi. Suprapubic pain
ii. New or marked increase in incontinence
vii. Gross hematuria
iii. New or marked increase in urgency
iv. New or marked increase in frequency
viii. Swelling, or tenderness of the testes, epididymis, or prostate
ix. New-onset of acute costovertebral angle pain or tenderness

v. New or marked increase in urinary retention $\quad x$. Episode of autonomic dysreflexia (with no other apparent cause)

NOTE: Only after clinical assessment and ruling-out of other possible causes should changes in mental status and functional decline, and sudden fever, rigors or newonset hypotension suggest UTI in patients; use clinical judgment. ${ }^{(3)}$

For Geriatric and Spinal Cord Injury (including conus/cauda equina): UTI may present atypically; use clinical assessment to guide decision for urine culture \& urinalysis.

\begin{tabular}{|c|c|c|c|c|}
\hline & $\sqrt{7}$ & Yes & \multirow{4}{*}{$\begin{array}{l}\text { Remove catheter (if possible) and reassess in } 24 \\
\text { hrs, or replace catheter before urine collection } \\
\text { unless contraindicated (e.g., catheter placed by } \\
\text { Urology, urethral stricture/trauma, patient } \\
\text { unable to tolerate procedure) }\end{array}$} \\
\hline & & Presence of indwelling catheter? & & \\
\hline & & $\downarrow$ No & & \\
\hline $\begin{array}{l}\text { Obtain urine for urinalysis (UA) }{ }^{\alpha} \text { looking for } \\
\text { leukocyte esterase (LE) and nitrites (NIT) }{ }^{(4)}\end{array}$ & AND & Obtain urine for urine culture & & \\
\hline
\end{tabular}

\section{LE (-) (i.e. Urine WBC 0-5/hpf) and/or NIT (-) \\ [or "WBC/bacteria not detected"] ${ }^{\beta}$}

UTI is unlikely. ${ }^{(4)}$

Consider alternate diagnosis.
LE (+) (i.e. Urine WBC >5/hpf) and/or NIT (+)

[or "WBC/bacteria detected"] ${ }^{\beta}$

\section{Special considerations:}

${ }^{\alpha}$ UA can be ordered alone for screening purposes.

A Applicable to Vancouver Acute sites only. Urine cultures will be reported only if urinalysis results are positive, unless discussed with Medical Microbiology.

UTI is possible: Presence of additional symptoms? -Fever, ${ }^{(2)}$ costovertebral angle tenderness, newonset hypotension, ${ }^{(3)}$ or signs of sepsis (refer to Sepsis Pre-printed Order)

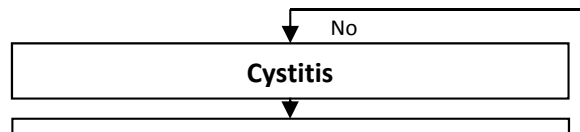

If symptoms are mild, may wait for culture results.

\section{$\checkmark$}

Review past culture results for antibiotic quidance

Preferred agents:

- Nitrofurantoin 50-100 mg QID or

Nitrofurantoin long acting (MacroBID ${ }^{\circledR}$ ) $100 \mathrm{mg} \mathrm{BID}$ if $\mathrm{CrCl} \geq 40 \mathrm{~mL} / \mathrm{min}$

[for mild cystitis only]

Co-trimoxazole 1 DS tab BID

Other options:

- Amoxicillin-clavulanate 500/125 mg TID

- Tetracycline 250-500 mg QID

- Fosfomycin $3 \mathrm{~g} \times 1$ dose

(Restricted use; contact Medical Microbiology)

If $\mathrm{PO}$ route not possible:

- CeFAZolin $1 \mathrm{~g}$ IV Q8H

- Tobramycin $4 \mathrm{mg} / \mathrm{kg}$ IV/IM Q24H if $\mathrm{CrCl} \geq 60 \mathrm{~mL} / \mathrm{min}$

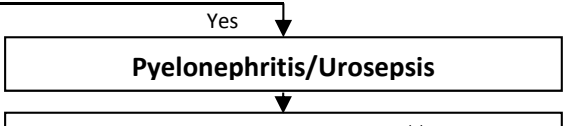

Obtain blood cultures $\times 2^{(5)}$

Consider renal ultrasound or CT (if indicated)

\begin{tabular}{|l|}
\hline Review past culture results for antibiotic guidance \\
Preferred PO agents for mild disease \\
- ceFURoxime $500 \mathrm{mg}$ TID \\
- Amoxicillin-clavulanate $500 / 125 \mathrm{mg}$ TID \\
- Co-trimoxazole $1 \mathrm{DS}$ tab BID \\
- Ciprofloxacin $500 \mathrm{mg} \mathrm{BID}$ \\
Preferred IV agents (Step-down to PO if possible - see below): ${ }^{(6,10)}$ \\
- CefTRIAXone $2 \mathrm{~g}$ IV Q24H \\
- If known or suspected Enterococcus, \\
ADD ampicillin $2 \mathrm{~g}$ IV Q6H \\
If significant beta-lactam allergy: \\
- Tobramycin 4-6 mg/kg IV Q24H if CrCl $\geq 60 \mathrm{~mL} / \mathrm{min}$ (use with caution in \\
$\quad$ elderly) \\
- If known or suspected Enterococcus, \\
ADD vancomycin (20 mg/kg) IV load, then $15 \mathrm{mg} / \mathrm{kg}$ Q12H \\
If severely ill/septic (refer to Sepsis Pre-printed Order): \\
- Piperacillin-tazobactam 3.375 g IV Q6H \\
If known or suspected resistance to above (e.g. post prostate biopsy): \\
- Meropenem $500 \mathrm{mg}$ IV Q6H
\end{tabular}

\section{Direct and Tailor Therapy}

- Select antibiotic with narrowest spectrum

based on culture results.

- Step-down to PO agent when appropriate.

- Assess clinical status; lack of improvemen

should prompt investigations for alternate

cause.

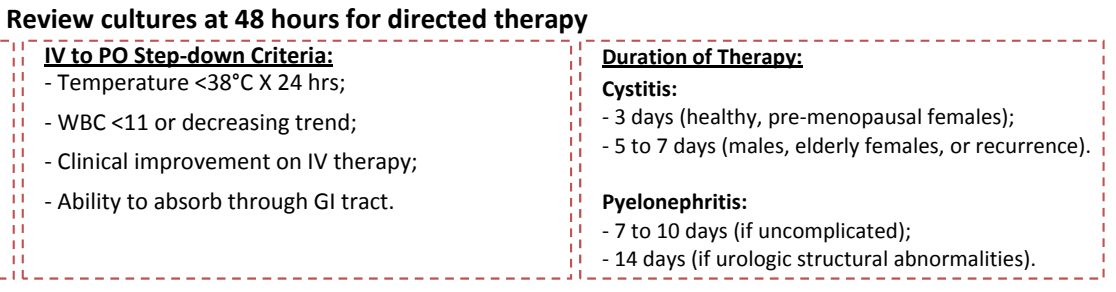

ASPIRES 
challenging [6]. Based on these challenges, there is an increased risk for specimen collection and treatment of asymptomatic patients.

The Antimicrobial Stewardship Programme: Innovation, Research, Education and Safety (ASPIRES) has been part of the Vancouver Coastal Health Authority's (VCH) Quality and Patient Safety department since 2012. One of the many contributions made by the ASPIRES team was the development of the Urinary Tract Infections Management Algorithm, which provides a systematic approach in the assessment and treatment of UTIs in non-pregnant adults, as seen in Figure 1.

As urine testing often drives prescribing, a key component of antibiotic stewardship around UTIs must be in the ordering and interpretation of urine tests. In the absence of a standardized approach, there is an increased risk of over-collection of samples and treatment of asymptomatic patients. Interpretation of the significance of the bacterial culture results requires evaluation of a number of factors, including clinical signs and symptoms, urinalysis results, specimen collection and storage, and antibiotic treatment $[7,9]$.

Urinalysis specimens provide critical information for interpretation of results and should be collected in coordination with urine for culturing $[4,8,10]$. According to recent studies, the minimum laboratory evaluation for a suspected UTI should include urinalysis for determination of leukocyte esterase (LE) and nitrate (NIT) levels by use of dipstick and microscopic evaluation for white blood cells. If the urinalysis is negative for LEs and NIT, a positive culture is very unlikely $[6,10,11]$.

To prevent contamination, urine specimens should be collected using a clean-catch midstream technique or by intermittent catheter. The clean-catch midstream approach is influenced by the patient's physical ability to perform the task and adhere to the instructions provided by the healthcare worker. Specimens should not be collected while using a bedpan, as they are not sterile and there is risk for urine to contact the surrounding perineum, resulting in contamination. Indwelling catheters must be replaced prior to obtaining specimens, as the inner and outer surfaces of the lumen develop a biofilm within days of insertion. Once a biofilm has been established, it protects the uropathogens from antimicrobial treatment and provides an environment that supports bacterial growth [8].

With respect to storage, urine specimens should be kept refrigerated and transported to the microbiology lab without delay. A delay in processing can lead to inaccurate results due to bacterial growth [10]. This is particularly pertinent to Powell River General Hospital (PRGH), as the Microbiology department was recently closed. Since June 2014, all urine specimens for culturing have been sent to the microbiology laboratory in Vancouver General Hospital (VGH) for processing. Urinalysis specimens continue to be processed at PRGH.

Although the ASPIRES algorithm outlines a systematic approach for the assessment and treatment of UTIs from the evaluation of clinical signs and symptoms, specimen collection, and treatment, the primary objective of this quality review was to assess the use of urinalysis in the diagnostic assessment of patients for UTIs.

\section{METHODS}

The quality review was undertaken in PRGH, a small community hospital located on the northern Sunshine Coast of British Columbia. PRGH is a 33-bed facility that supports a regional district with an estimated population size of 20,000. The annual number of admissions for 2015-2016 was 1,599, with an average length of stay of 6.89 patient days. Older adults ( $\geq 65$ years) accounted for 819 (51\%) of the admissions, with an average length of stay of 8.7 patient days.

\section{Design}

The review involved two separate components: a staff educational survey and a retrospective analysis of electronic microbiology data.

Ethical review was not required for this quality assurance project as it involved evaluation of expected knowledge and practice.

\section{Staff survey}

The objective of the staff survey was to evaluate the interdisciplinary team's knowledge of urine specimen collection and interpretation of results, which would identify opportunities for improvement. The survey was distributed during the week of March 13 to March 17, 2017 to 19 staff members working day shifts on the medical/surgical unit. 17 of the 19 staff were nurses (LPN/RN), one staff member was an occupational therapist, and one was a physiotherapist. Completed surveys were returned and results were entered into an Excel spreadsheet.

The survey consisted of 16 items based on the $\mathrm{VCH}$ Professional Practice guidelines Indwelling urinary catheter: Guideline to prevent catheter associated urinary tract infections (CAUTI) - Adult and the ASPIRES algorithm VCH Management of Urinary Tract Infections in Non-pregnant Adults. Survey items 1 to 7 evaluated sample collection; items 8 to 16 evaluated the interpretation of urine specimen results.

\section{Electronic data review}

Microbiology data, including the date and time of urine culture specimen results, was extracted for all patients admitted to the medical/surgical unit over a one-year period (September 1, 2015 to August 31, 2016). Corresponding urinalysis data was retrieved manually from the local electronic patient care information system.

Data was imported into Statistical Package for Social Sciences (SPSS, version 18) and reviewed for data quality prior to conducting the descriptive and statistical analyses.

\section{Definitions}

For the purpose of analysis, the following case definitions were used.

\section{Urinalysis}

"Urinalysis test" was defined as a physical, chemical, or microscopic analysis or examination of urine. It determines the levels of LEs and NITs by use of a dipstick and a microscopic examination for white blood cells. 
"Positive urinalysis" was defined as a urine specimen resulting in LEs and/or NITs of any value, whereas a "negative urinalysis" was defined as a urine specimen resulting in the absence of LEs (-) and NITs (-).

\section{Urine cultures}

"Positive urine culture with significance" was defined as a single urine specimen with the isolation of one predominate bacterial strain with a count of $>100$ million CFU/L [4, 8]. "Negative urine cultures" included those with no growth, an insignificant quantity of growth, or mixed growth due to probable contaminates.

\section{RESULTS}

\section{Education survey}

All 19 surveys distributed were returned for a response rate of $100 \%$. No survey items were left blank. There was a general consensus overall: staff scored higher on Section B (interpretation of results) at $80.7 \%$ than on Section A (specimen collection) at $69.2 \%$, as summarized in Table 1 . The difference was statistically significant (Fisher's exact test, $p=0.02$ ). Four questions were answered with less than 54\% accuracy and were distributed between both sections. The question that received the lowest number of correct answers was A7 (16\%), which measured knowledge on urine collection methods for the catheterized patient.

\section{Microbiology data}

Sample characteristics

318 urine specimens were collected from September 1, 2015 to August 31, 2016 and submitted to the VGH microbiology laboratory for culturing, as noted in Figure 2. 148 (46.5\%) specimens were collected from males and 170 (53.5\%) specimens were collected from females. The mean age was 73.6 years, with a median of 77 years and a range of 18 to 101 years. Statistical analysis using a non-parametric median test showed that the difference in the median age for males (78.5) vs females (77.0) was not statistically significant $(p=0.742)$.

As noted in Table 2, 62 (19.5\%) of the 318 specimens did not have a corresponding urinalysis. Of the 256 urinalysis specimens processed, 136 (53.1\%) were negative for both LEs and NITs,

\section{FIGURE 2: Distribution by age.}

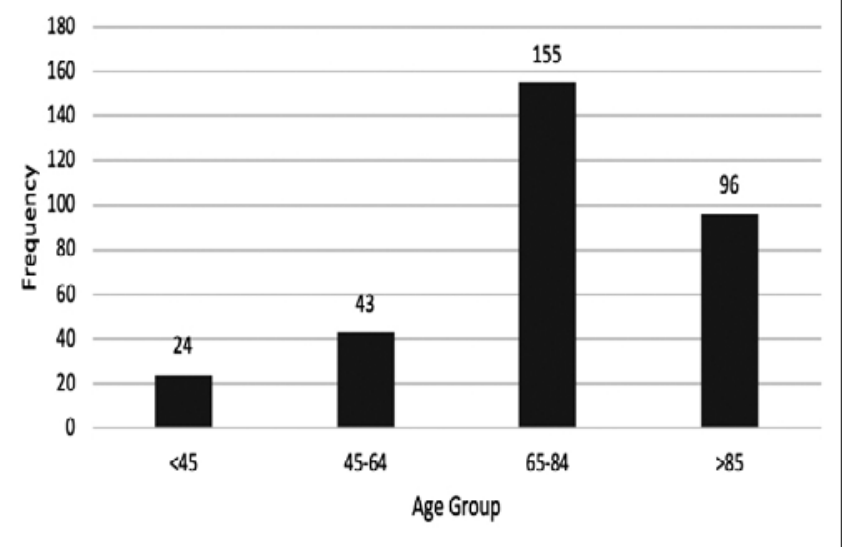

and 120 (46.9\%) were positive by definition. 78 (24.5\%) of the 318 urine cultures met microbiologic criteria for significant growth of organisms and were considered to be positive urine cultures; of these, 50 (64.1\%) corresponded to a positive urinalysis, in comparison to nine $(11.5 \%)$ corresponding to negative urinalysis. The remaining 19 (24.5\%) positive cultures were associated with an absent or missing urinalysis.

184 (57.9\%) of the 318 specimens resulted in no growth, of which $45(24.4 \%)$ were associated with a positive urinalysis and 105 (57.1\%) were associated with a negative urinalysis. The remaining 34 (18.5\%) specimens, which had no growth, were associated with a missing urinalysis. 56 (17.6\%) of the 318 cultures submitted did not grow organisms with numbers greater than 100 million CFU/L and were not considered to be positive based on our case definition.

There was a highly statistically significant relationship between urinalysis and clinical significance (Fisher's exact test $p<0.0001)$. Specifically, a positive urine culture (> 100 million CFU/L) was statistically significantly more likely to be observed when there was a positive urinalysis, and a negative culture ( $<100$ million CFU/L and No growth) was statistically significantly more likely with a negative urinalysis.

\section{TABLE 1: Educational survey.}

\begin{tabular}{|c|c|c|}
\hline Question & Correct & Percentage \\
\hline $\begin{array}{l}\text { A1 Elderly population and risk } \\
\text { factors for developing a UTI. }\end{array}$ & 17 & $89 \%$ \\
\hline $\begin{array}{l}\text { A2 Change in cognitive function - } \\
\text { indication of a UTI. }\end{array}$ & 18 & $95 \%$ \\
\hline $\begin{array}{l}\text { A3 Malodorous/cloudy urine - } \\
\text { indication of a UTI. }\end{array}$ & 9 & $47 \%$ \\
\hline A4 Signs and symptoms of a UTI. & 17 & $89 \%$ \\
\hline $\begin{array}{l}\text { A5 Collection of a urinalysis } \\
\text { specimen. }\end{array}$ & 14 & $74 \%$ \\
\hline A6 Ideal urine specimen for culture. & 14 & $74 \%$ \\
\hline $\begin{array}{l}\text { A7 Urine specimen from a } \\
\text { catheterized patient. }\end{array}$ & 3 & $16 \%$ \\
\hline Section A subtotal & & $69.1 \%$ \\
\hline $\begin{array}{l}\text { B8 Urinalysis results and } \\
\text { interpretation. }\end{array}$ & 18 & $95 \%$ \\
\hline B9 UTI vs asymptomatic bacteriuria. & 15 & $79 \%$ \\
\hline $\begin{array}{l}\text { B10 Definition of a positive urine } \\
\text { culture. }\end{array}$ & 10 & $53 \%$ \\
\hline $\begin{array}{l}\text { B11 Prevalence of bacteriuria in } \\
\text { elderly patients. }\end{array}$ & 17 & $89 \%$ \\
\hline $\begin{array}{l}\text { B12 Treatment of a positive culture } \\
\text { in asymptomatic patients. }\end{array}$ & 15 & $79 \%$ \\
\hline $\begin{array}{l}\text { B13 Asymptomatic bacteriuria vs a } \\
\text { UTI. }\end{array}$ & 16 & $84 \%$ \\
\hline $\begin{array}{l}\text { B14 Treatment of asymptomatic } \\
\text { bacteriuria. }\end{array}$ & 18 & $95 \%$ \\
\hline B15 Location of urinalysis results. & 19 & $100 \%$ \\
\hline $\begin{array}{l}\text { B16 Location of urine cultures } \\
\text { results. }\end{array}$ & 10 & $53 \%$ \\
\hline Section B subtotal & & $80.8 \%$ \\
\hline
\end{tabular}




\begin{tabular}{|c|c|c|c|c|c|}
\hline \multirow{2}{*}{\multicolumn{2}{|c|}{ Urinalysis }} & \multicolumn{3}{|c|}{ Culture Significance } & \multirow{3}{*}{$\begin{array}{r}\text { Total } \\
62\end{array}$} \\
\hline & & \multirow{2}{*}{$\begin{array}{c}\text { Greater than } 100 \\
\text { million CFU/L } \\
19 \\
\end{array}$} & \multirow{2}{*}{$\begin{array}{l}\text { Less than } 100 \\
\text { million CFU/L } \\
9\end{array}$} & \multirow{2}{*}{$\begin{array}{c}\text { No growth } \\
34\end{array}$} & \\
\hline Not collected & Count & & & & \\
\hline & Column \% & $24.4 \%$ & $16.1 \%$ & $18.5 \%$ & \\
\hline (-) Leukocyte and (-) Nitrate & Count & 9 & 22 & 105 & 136 \\
\hline & Column \% & $11.5 \%$ & $39.3 \%$ & $57.1 \%$ & \\
\hline$(+)$ Leukocyte and/or (+) Nitrate & Count & 50 & 25 & 45 & 120 \\
\hline & Column \% & $64.1 \%$ & $44.6 \%$ & $24.4 \%$ & \\
\hline Total & Count & 78 & 56 & 184 & 318 \\
\hline
\end{tabular}

The organisms isolated in urine cultures with significance greater than 100 million CFU/L are summarized in Figure 3. The predominant individual organisms identified were Escherichia coli $(\mathrm{n}=23 ; 29.5 \%)$, and Enterococcus faecalis $(\mathrm{n}=13 ; 16.7 \%)$. Mixed organisms contributed the secondhighest percentage (21.8\%).

\section{FIGURE 3: Distribution by organism.}

Group B beta hemolytic Streptococcus

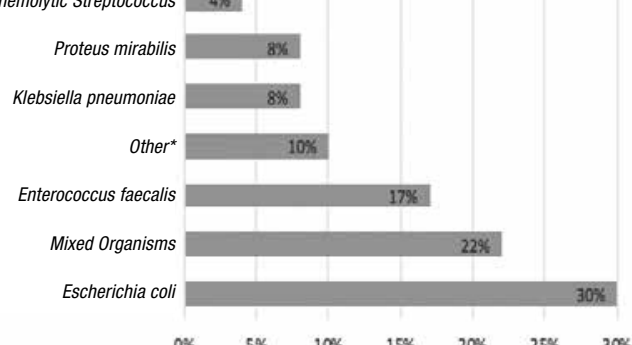

* Other includes organisms with a culture significance less than or equaling 1.3\%: Coagulase negative staphylococci; Escherichia coli and Klebsiella pneumoniae; Klebsiella pneumoniae and Enterococcus faecalis; Proteus vulgaris; Pseudomonas aeruginosa and Candida albicans; and Staphylococcus aureus.

\section{DISCUSSION}

The ASPIRES algorithm provides a systematic approach in the assessment, diagnosis, and treatment of UTIs. Findings from this quality assurance review reinforce the importance of adherence to evidence-driven best practices to ensure consistently highquality care for our patients. Results revealed areas where concordance with the guidelines needs to be improved.

Firstly, the practical and effective use of a urinalysis specimen in combination with a urine culture in the assessment and diagnosis of a UTI needs to be recognized. Evidence shows that specimens resulting in a negative urinalysis are unlikely to be associated with a positive culture (less than 20\%) [7, 10, 11].

In this review, 62 (19.5\%) of the 318 cultures did not have a corresponding urinalysis. Of the 256 urinalysis specimens collected, 136 (53.1\%) resulted in a negative urinalysis and of those, $105(77.2 \%)$ were associated with no growth. These results correspond to what is reported in the literature and support the intent of the ASPIRES algorithm to reduce unnecessary testing and treatment of urine cultures, which can lead to significant side effects and expense and can drive antibiotic resistance $[2,6,10]$.

As with many rural hospitals affected by the centralization of services, the local microbiology laboratory has been closed, impacting the processing of urine specimens. In response, two separate workflows have evolved to manage urine specimens. All urine for culture and sensitivity is sent to the regional laboratory irrespective of an absent or negative urinalysis. This workflow limits the ability of front-line staff to be compliant with the algorithm and results in an increased number of urine specimens sent for culturing.

Secondly, opportunities for improvement in staff knowledge and practice specific to specimen collection became apparent with the high proportion of cultures that resulted in mixed organisms, which is suggestive of contamination. In this review, mixed organisms accounted for $22 \%$ of all organisms with a culture of significance. This was second to $E$. coli, which was the most predominate organism. "Mixed growth" is defined as the presence of more than one bacterium in the urine sample $[8,9]$. Cultures that contain more than one organism are usually considered contaminated. Contamination occurs when samples are not collected properly or when there is a substantial delay in processing. Mixed organisms do not meet the clinical definition of a positive culture and therefore impede diagnosis, resulting in a delay of treatment and/or inappropriate antimicrobial use [8, 9, 12].

The challenges of midstream urine collections without contamination are greatly underestimated. Fecal and urinary incontinence have an impact on specimen collection and may play a significant role in contamination. Further investigation is required to assess urine specimen collection techniques, as it is unclear if staff were able to provide adequate instructions to patients in the correct methods of proper cleansing prior to obtaining a specimen.

The results of the staff survey provided insight into the nurses' knowledge of sample collection in the presence of an indwelling catheter. Only $16 \%$ of the staff surveyed recognized the need to replace the catheter prior to obtaining urine for culturing. This may have contributed to the large proportion of specimens that resulted in mixed organisms. 
Another contributing factor that may influence contamination is the extended period of time between collection of the specimen on the unit and the processing of the urine culture in the regional laboratory.

Lastly, the diagnosis of symptomatic UTIs in older adults is challenging, as most accepted definitions require the presence of localized genitourinary symptoms. However, underlying medical comorbidities such as dementia and stroke may impair peoples' ability to communicate symptoms. As a result, clinicians rely on nonspecific symptoms such as a change in behaviour and laboratory data for diagnosis [2, 6, 13, 14].

A disproportionate number of urine cultures $(n=251 ; 78.9 \%)$ were collected from adults ( $\geq 65$ years), suggesting that older adults were more likely to be investigated for possible UTIs. This may be attributed to both the increased risk of UTI for older adults as well as the challenges associated with diagnosis $[2,14-16]$. As a chart review was not completed as part of the review, it is unclear if urine specimen collection was influenced by a change in patient behaviour or based on signs and symptoms. The survey suggests opportunity for education in the clinical assessment of a UTI, as only $47 \%$ of the participants answered the question pertaining to malodorous and cloudy urine correctly.

This quality review had limitations. Firstly, chart reviews were not conducted to verify the signs and symptoms associated with the urine specimen collection, nor did the review investigate the appropriateness of antimicrobial therapy. Urine specimens obtained from catheterized patients were not distinguished from non-catheterized patients, as this is not reliably documented in the electronic patient care information system.

In summary, older adults represent a large and growing population of hospitalized patients who are at high risk of complications during their stay. UTIs have been identified as the single most common healthcare-acquired infection [1, 2, 16, 17]. Studies have shown that the diagnosis of a symptomatic UTI is challenging due to chronic genitourinary symptoms and cognitive deficits among elderly patients. Urinalysis specimens can provide critical information for interpretation of results in conjunction with urine for culturing.

In the absence of a standardized approach, there is an increased risk of over-collection of samples and treatment of asymptomatic patients, which may lead to a variety of negative consequences, including the development of multidrug-resistant organisms [2].

\section{REFERENCES}

1. Nicolle, L. E. (2008). The prevention of hospital-acquired urinary tract infection. Clinical Infectious Disease, 46(2) 251-253. doi: $10.1086 / 524663$

2. Silver, S. A., Baillie, L., \& Simor, A. E. (2009). Positive urine cultures: A major cause of inappropriate antimicrobial use in hospitals? Canadian Journal of Infectious Diseases Medical Microbiology, 20(4), 107-111. Retrieved from https://www.ncbi.nlm.nih.gov/pmc/articles/PMC2807252/

3. Foxman, B. (2002). Epidemiology of urinary tract infections: Incidence, morbidity, and economic cost. Disease-a-Month, 49(2), 53-70. doi: https://doi.org/10.1067/mda.2003.7

4. Vancouver Coastal Health. (2013). ASPIRES urinary tract infection algorithm. Retrieved from http://vhpharmsci.com/Resources/Pocket/Antimicrobial-Stewardship-Programme-ASPIRES-UTI-Education-Slides\%20 (Feb\%202013).pdf

5. Soliman, Y., Meyer, R., \& Baum, N. (2016). Falls in the elderly secondary to urinary symptoms. Reviews in Urology, 18(1), 28-32. Retrieved from https://www.ncbi.nlm.nih.gov/pmc/articles/PMC4859925/

6. Rowe, T. A., \& Juthani-Mehta, M. (2013). Urinary tract infection in older adults. Aging Health, 9(5). doi: 10.2217/ahe.13.38

7. Vancouver Coastal Health (VCH). (2017). VCH Management of urinary tract infections (UTI) in Non-pregnant Adults. Retrieved from http://www. vhpharmsci.com/Resources/Pocket/VCH\%20UTI\%20Algorithm\%20 _24OCT15_\%20_FINAL_.pdf

8. Vancouver Coastal Health (VCH). (2017). Indwelling urinary catheter: Guideline to prevent catheter associated urinary tract infections (CAUTI) - Adult. Retrieved from http://shop.healthcarebc.ca/vch/ VCHDSTs/D-00-07-30110.pdf

9. Goering, R., Dockrell, H., Zuckerman, M., Wakelin, D., Roitt, I., Mims, C., \& Chiodini, P. (2008). Mims' medical microbiology (4th ed.). Philadelphia, PA: Elsevier.

10. Meyrier, A. (2015, July). Sampling and evaluation of voided urine in the diagnosis of urinary tract infection in adults. Retrieved from http://www. uptodate.com/contents/sampling-and-evaluation-of-voided-urine-in-thediagnosis-of-urinary-tract-infection-in-adult?source $=$ search result + searc $\mathrm{h}+\mathrm{UTI}+$ and + urine+sampling + selectTitle $=1 \% 7 \mathrm{E} 150$

11. Ouslander, J. G., Schapira, M., Fingold, S., \& Schnelle, J. (1995). Accuracy of rapid urine screen tests among incontinent nursing home residents with asymptomatic bacteria. Journal of the American Geriatrics Society, 43(7), 772-775.

12. Bekeris, L. G., Jones, B. A., Walsh, M. K., \& Wagar, E. A. (2008). Urine culture contamination: A College of American Pathologists Q-Probes study of 127 laboratories. Archives of Pathology \& Laboratory Medicine, 132(6), 913-917. doi: 10.1043/1543-2165(2008)132[913:UCCACO]2.0 .CO;2

13. Detweiler, K., Mayers, D., \& Fletcher, S. (2015). Bacteriuria and urinary tract infections in the elderly. Urologic Clinics of North America, 42(4), 561-568. doi: 10.1016/j.ucl.2015.07.002

14. Daley, P., Penney, C., Wakeham, S., Compton, G., McKim, A., O'Keefe, J., Barrett, B., \& Nicolle, L. (2015). Urinary tract infection diagnosis and response to therapy in long-term care: A prospective observational study. Canadian Journal of Infectious Diseases and Medical Microbiology, 26(3), 133-136. Retrieved from https://www.ncbi.nlm.nih.gov/pmc/articles/ PMC4507838/

15. Fekete, T., \& Hooton, T. M. (2016, August). Approach to the adult with asymptomatic bacteriuria. Retrieved from https://www.uptodate.com/ contents/approach-to-the-adult-with-asymptomatic-bacteriuria

16. Rowe, T. A., \& Juthani-Mehta, M. (2014). Diagnosis and management of urinary tract infection in older adults. Infectious Disease Clinics of North America, 28(1), 75-89. doi: 10.1016/j.idc.2013.10.004

17. Nicolle, L. E. (2016). Urinary tract infections in the older adult. Clinics in Geriatric Medicine, 32(3), 523-538. doi: 10.1016/j.cger.2016.03.002 * 